\title{
Orchidaceae no município de Vitória da Conquista, Bahia: lista de espécies e similaridade florística en- tre áreas da Bahia e Minas Gerais
}

\author{
Orchidaceae in the municipality of Vitória da Conquista, Bahia: species list and floristic \\ similarity among areas in Bahia and Minas Gerais
}

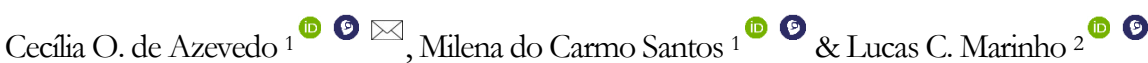

1. Universidade Estadual do Sudoeste da Bahia, Departamento de Ciências Naturais, Estrada do Bem-Querer, Vitória da Conquista, Bahia, Brasil

2. Sítio 2 Irmãos, Porto Seguro, Bahia, Brasil

3. Universidade Federal do Maranhão, São Luís, Maranhão, Brasil

Palavras-chave:

Flora da Bahia. Floresta Estacional Decidual Montana. Mata de cipó. Orquídeas. Planalto de Conquista.

\section{Keywords:}

Flora of Babia. Deciduous seasonal forest. Liana forest. Orcbids. Plateau of Conquista.

Recebido em: 29/03/2021

Aceito em: 17/05/2021

Editor responsável: Jaílson S. de Novais (UFSB)

eISSN: 2595-6752

\section{(c) (i)}

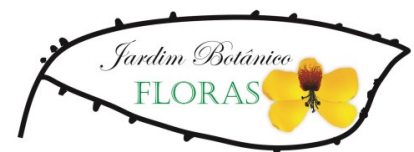

\section{Resumo}

Aqui, apresentamos o levantamento das Orchidaceae de Vitória da Conquista, Brasil, e uma análise da similaridade florística entre dez áreas da Bahia e Minas Gerais. Foram encontradas no município 44 espécies de orquídeas, sendo duas novas ocorrências para o Nordeste brasileiro, Acianthera panduripetala e Specklinia bymenantha, além de uma nova ocorrência para o estado da Bahia, Pabstiella seriata. Foram conduzidas análises utilizando o algoritmo UPGMA, coeficientes de Jaccard e Dice, e análise de correspondência. A análise de agrupamento revelou baixos índices de similaridade, exceto para Serra Negra + Parque Estadual do Ibitipoca. Dois grupos se formaram: um de áreas de Florestas Secas e outro grupo que inclui as áreas de Campos Rupestres e de Floresta Ombrófila dos dois estados. Os resultados sugerem que as áreas de mesma fitofisionomia estão mais relacionadas entre si do que com a vegetação do mesmo estado.

\section{Abstract}

Here we present a survey of Orchidaceae from Vitoria da Conquista, Brazil and an analysis of the floristic similarity among ten areas in Babia and Minas Gerais. Fourty-four species of orchids were found in the municipality. Among the species found, two are new records for the Northeast region of Brazil, Acianthera panduripetala and Specklinia bymenantha, and one is a new occurrence for the state of Bahia, Pabstiella seriata. Analyzes were conducted using the UPGMA algorithm, Jaccard and Dice coefficient and Correspondence Analysis. The cluster analysis revealed low similarity rates, except for Serra Negra + Ibitipoca State Park. Two groups were formed: one group of dry forest areas and another group encompassing the Rupestrian Fields and Ombrophilous Forest areas of the two states. The results suggest that the areas of the same phytophysiognomy are more related to each other than to the vegetation of the same state.

\section{Introdução}

O município de Vitória da Conquista localiza-se no Planalto de Conquista, região conhecida geopoliticamente como Sudoeste baiano. A vegetação predominante no Planalto de Conquista é regionalmente conhecida como mata-de-cipó - ou floresta de liana -, um tipo de Floresta Estacional Decidual Montana que possui na sua composição, além de elementos característicos da Caatinga, forte influência da Floresta Atlântica (Andrade-Lima, 1971) e da alta umidade resultan- 
te da sua altitude (Queiroz et al., 2006), que varia de 600 a $1.100 \mathrm{~m}$. Estas características conferem à região uma vegetação única, com áreas mais úmidas e árvores de grande porte próximas aos cursos d'água (e.g. Reserva do Poço Escuro - Marinho; Azevedo, 2013), áreas secas com lianas robustas e árvores baixas (e.g. área da matinha na Universidade Estadual do Sudoeste da Bahia - Goldenberg et al., 2020), e manchas de afloramento rochoso, composto por cascalho de quartzo quase puro e flora constituída apenas por uma vegetação arbustiva baixa em áreas de maior altitude (e.g. Serra do Periperi - Taylor, 1992).

Apesar da singularidade florística do município, pouco se conhecia sobre sua flora. Nos últimos dez anos, uma série de novas ocorrências vêm sendo registradas a partir de espécimes coletados em Vitória da Conquista, não apenas para a Bahia, mas também para o Nordeste do país e para o Brasil, como por exemplo: os cinco novos registros de algas continentais para o estado da Bahia e duas variedades de algas, Cosmarium clepsydra Nordst var. dissimile (Raciborski) Krieger \& Gerloff e C. tenue Archer var. depressum Irenee-Marie para o Brasil (Santos et al., 2013); o musgo Syrrhopodon lycopodioides (Brid.) Müll. Hal. foi citado pela primeira vez para a região Nordeste do Brasil (Souza et al., 2015); Dittrich e Souza (2013) também encontraram em Vitória da Conquista uma samambaia aquática, Azolla microphylla Kaulf. (Salviniaceae), sendo este o primeiro registro para o estado da Bahia. Além de dois novos registros de Orchidaceae para o Nordeste do Brasil: Acianthera saurocephala (Lodd.) Pridgeon \& M.W.Chase (Marinho; Azevedo, 2011) e Cyclopogon variegatus Barb.Rodr. (Azevedo; Marinho, 2012); e 11 novos registros de solanáceas para o município de Vitória da Conquista (Moura; Caires, 2021).

Além das novas ocorrências em diversos grupos vegetais, novas espécies também têm sido descritas com base em espécimes provenientes do município de Vitória da Conquista, como: Ficus caatingae R.M.Castro (Moraceae - Castro; Rapini, 2006); Quesnelia conquistensis Leme (Bromeliaceae, Leme, 2008); Aechmea avaldoana Leme \& W.Till (Bromeliaceae - Leme et al., 2014); Pabstiella acrogenia Luer \& Toscano e P. quasi Luer \& Toscano (Orchidaceae Luer; Toscano de Brito, 2011); Magnolia brasiliensis C.O.Azevedo et al. (Magnoliaceae - Azevedo et al., 2018) e Miconia babiana R.Goldenb. \& J.Coelho (Melastomataceae, Goldenberg et al., 2020). A maioria destas espécies é conhecida apenas de uma ou poucas localidades, o que as tornam raras.

As particularidades florísticas do município de Vitória da Conquista levantam questões não apenas sobre as espécies que compõem as florestas da região, mas também sobre suas afinidades florísticas dentro e fora do estado. A elevação altitudinal do Planalto de Conquista e a ocorrência de algumas espécies tipicamente relacionadas à Chapada Diamantina [e.g. Aristolochia melastoma Silva Manso ex Duch [Abreu; Giulietti, 2016]; Aspilia almasensis D.J.N.Hind (Alves; Roque, 2016); Jacaranda caroba (Vell.) DC. [SilvaCastro et al., 2007]; Bonnetia stricta (Nees) Ness \& Mart., L.C. Marinho, obs. pes.] sugerem uma possível aproximação na composição florística entre as áreas. Por outro lado, a proximidade geográfica e a presença de manchas de Florestas Estacionais Semideciduais em algumas áreas de Minas Gerais podem conduzir a um resultado diferente. Aqui, nós propomos listar as espécies de Orchidaceae do município de Vitória da Conquista e investigar suas relações florísticas com áreas da Bahia e de Minas Gerais.

\section{Material e Métodos}

\section{Dados de distribuição}

A lista de espécies de Vitória da Conquista foi confeccionada por meio de coletas realizadas desde 2008 em diversas áreas do município e informações adicionais dos autores. Algumas destas expedições fizeram parte de trabalhos florísticos em áreas específicas (a saber, Marinho; Azevedo, 2011; Azevedo; Marinho, 2012; Marinho; Azevedo, 2013; Santos, 2013). Os espécimes coletados foram incorporados aos herbários HVC e HUESBVC com duplicatas enviadas ao herbário HUEFS (siglas de acordo com Thiers, 2021, continuamente atualizado).

Babia - O check list das Orchidaceae da Chapada Diamantina (Smidt; Toscano de Brito, 2005) foi utilizado como base para a lista desta região, com algumas alterações e inclusões observando outros trabalhos publicados para a área (Harley; Simmons, 1986; Toscano de Brito, 1995; 1998; Toscano de Brito; Queiroz, 2003; Azevedo; van den Berg, 2007; Bastos; van den Berg, 2012a; Vieira et al., 2014). Para a região de Boa Nova foi utilizada a Sinopse das Orchidaceae do Parque Nacional de Boa Nova, uma importante área de transição entre a Caatinga e a Floresta Atlântica (Rêgo; Azevedo, 2017). A lista do Sul da Bahia foi organizada compilando os dados de três publicações (Amorim et al., 2009; Coelho; Amorim, 2014; Leitman et al., 2014), que abrangem quatro áreas de Floresta Atlântica, situadas nos municípios de Almadina (Serra do Corcovado Coelho; Amorim, 2014), Barro Preto (Reserva Serra da Pedra Lascada - Amorim et al., 2009; Coelho; Amorim, 2014; Leitman et al., 2014), Arataca/São José da Vitória (Reserva Serra das Lontras Amorim et al., 2009; Leitman et al., 2014) e Camacan (Reserva Particular do Patrimônio Natural Serra Bonita - Amorim et al., 2009; Leitman et al., 2014), todos no sul do estado.

Minas Gerais - Para a lista das áreas de Campo Rupestre de Minas Gerais foram utilizadas a Flora da Serra do Cipó (Barros, 1987) e a Flora de Grão-Mogol (Barros; Pinheiro, 2004). A lista da Mata do Baú seguiu Menini Neto et al. (2004b), representando uma área de transição entre Floresta Estacional Semidecidual e Campocerrado (Menini Neto et al., 2004b). A lista de espécies das Serras Negra e do Funil segue Abreu et al. (2011) e representa uma área de Floresta Atlântica, sendo caracterizada por apresentar um mosaico composto por formações campestres e florestais (Abreu et al., 2011). As espécies de orquídeas do Parque Estadual de Ibitipoca teve como base Menini Neto et al. (2007), representando outro mosaico de formações vegetais, das quais o Campo Rupestre ocupa a maior extensão, sendo também representado por diversas formações florestais, como Floresta Estacional Semidecidual Montana e Floresta Ombrófila Densa Altimontana (Menini Neto et al., 2007). Para a lista de espécies da Reserva Biológica da Represa do Grama, utilizamos a lista fornecida por Menini Neto et al. (2004a). Esta área é caracterizada por apresentar uma fitofisionomia de Floresta Estacional Semidecidual (Menini Neto et al., 2004a). 


\section{Análises dos dados}

Para a análise de agrupamento, os seguintes critérios foram aplicados: espécimes identificados apenas até gênero foram removidos quando outras espécies do gênero já constavam na lista. Espécies que constavam como of. (identificação a conferir) ou aff. (espécie afim) em uma área e identificadas em outra área foram consideradas co-específicas. Os nomes de espécie foram uniformizados, atualizados e os sinônimos nomenclaturais excluídos de acordo com a Flora do Brasil (2021, em construção).

Para a análise comparativa foram compiladas informações de dez regiões (Tabela 1) em uma matriz com dados de presença e ausência. As análises foram realizadas no software PAST (Paleontological Statistics) Versão 4.03 (Hammer et al., 2001). Foram utilizados os coeficientes de similaridade de Jaccard e de Dice, os quais atuam de maneira semelhante (Albuquerque et al., 2016), mas com a atribuição de um maior peso às espécies compartilhadas no coeficiente de Dice. A partir destes resultados foi feita uma análise de agrupamento das dez áreas da Bahia e de Minas Gerais, utilizando o algoritmo UPGMA (Unweighted Pair-Group Method using Arithmetic Averages) para agrupamento (Sneath; Sokal, 1973). O suporte interno dos grupos foi calculado utilizando 5.000 replicações de bootstrap. Além destas, uma análise de correspondência (Correspondence Analysis - CA; Davis, 1986) foi realizada com o intuito de medir o grau de associação das áreas e facilitar a interpretação dos conjuntos por meio de um modelo gráfico.

\section{Resultados e Discussão}

Foram encontradas até o momento 44 espécies de Orchidaceae, distribuídas em 30 gêneros no município de Vitória da Conquista (Tabela 2). Os gêneros com maior número de espécies foram: Acianthera Scheidw. (Figura 1a), Campylocentrum Benth. (Figura 1e) e Gomesa R.Br. (Figura 2c), com quatro espécies, Encyclia Hook. (Figura 2a) e Pabstiella Brieger \& Senghas (Figura 2h), com três espécies cada, seguidos de V anilla Mill. (Figura 3e), com duas espécies cada. Entretanto, a maior parte dos gêneros encontrados no município está representada por apenas uma espécie (Tabela 2).

Duas novas ocorrências para o Nordeste do Brasil são aqui registradas: Acianthera panduripetala (Barb.Rodr.) Pridgeon \& M.W.Chase (Figura 1a), até o momento conhecida apenas para as regiões Sudeste e Sul (Flora do Brasil, 2021, em construção), e Specklinia bymenantha (Lindl.) F.Barros \& V.T.Rodrigues (Figura 3b), que até o momento havia sido registrada apenas para o estado do Rio de Janeiro, em áreas de Floresta Ombrófila (Flora do Brasil, 2021, em construção). Além destas, Pabstiella seriata (Lindl.) Luer \& Toscano (Figura $2 \mathrm{~h}$ ) constitui-se em um novo registro para o estado da Bahia, coletada anteriormente no Nordeste (Paraíba), Sudeste (Espírito Santo, Rio de Janeiro, São Paulo) e Sul (Paraná, Santa Catarina) (Flora do Brasil, 2021, em construção). Dentre as espécies em gêneros já monografados para o projeto Flora da Bahia (Giulietti et al., 2006), Catasetum purum Nees \& Sinning (Figura 1g Bastos; van den Berg, 2012b) e Cattleya amethystoglossa Linden \&

Tabela 1. Relação das dez áreas adotadas na análise de similaridade florística de Orchidaceae, incluindo suas respectivas siglas, tipo vegetacional (bioma e ecossistemas predominantes na área), localidade, altitude, número de espécies (spp.), número de espécies exclusivas para cada área ( $\mathrm{N}^{\circ}$ spp. exclusivas) e referências bibliográficas para as respectivas fontes de dados. Para a distribuição geográfica das localidades veja a Fig. 4.

\begin{tabular}{|c|c|c|c|c|c|c|}
\hline Flora & Tipo vegetacional & Localidade & Altitude & Spp. & $\begin{array}{l}\mathrm{N}^{\circ} \text { spp. } \\
\text { exclusivas }\end{array}$ & Referências \\
\hline $\begin{array}{l}\text { Serra de Grão-Mogol } \\
\text { (GrM) }\end{array}$ & $\begin{array}{l}\text { Cerrado/Caatinga } \\
\text { Campo Rupestre }\end{array}$ & Grão-Mogol (MG) & $700-1.295$ & 30 & 12 & Barros; Pinheiro, 2004 \\
\hline $\begin{array}{l}\text { Chapada Diamantina } \\
\text { (ChD) }\end{array}$ & $\begin{array}{l}\text { Caatinga } \\
\text { Campo Rupestre }\end{array}$ & $\begin{array}{l}\text { Abaíra, Água Quente, } \\
\text { Andaraí, Jacobina, Lençóis, } \\
\text { Morro do Chapéu, Mu- } \\
\text { cugê, Palmeiras, Piatã, Rio } \\
\text { de Contas (BA) }\end{array}$ & $900-1.958$ & 178 & 70 & $\begin{array}{c}\text { Smidt; Toscano de Brito, } \\
\text { 2005; Harley; Simmons, } \\
\text { 1986; Toscano de Brito, } \\
\text { 1995; 1998; Toscano de } \\
\text { Brito; Queiroz, 2003; Aze- } \\
\text { vedo; van den Berg, 2007; } \\
\text { Bastos; van den Berg, 2012a; } \\
\text { Vieira et al., } 2014\end{array}$ \\
\hline Serra do Cipó (Cip) & $\begin{array}{c}\text { Cerrado } \\
\text { Campo Rupestre }\end{array}$ & Santana do Riacho (MG) & 1.200 & 80 & 29 & Barros, 1987 \\
\hline Sul da Bahia (SdB) & $\begin{array}{l}\text { Floresta Attântica } \\
\text { Floresta Ombrófila Densa }\end{array}$ & $\begin{array}{l}\text { Almadina, Arataca/São } \\
\text { José da Vitória, Barro } \\
\text { Preto, Camacan (BA) }\end{array}$ & $300-1.080$ & 158 & 75 & $\begin{array}{l}\text { Amorim et al., 2009; } \\
\text { Coelho; Amorim, 2014; } \\
\text { Leitman et al., } 2014\end{array}$ \\
\hline $\begin{array}{l}\text { Serra Negra e do } \\
\text { Funil (SNF) }\end{array}$ & $\begin{array}{l}\text { Floresta Atlântica } \\
\text { Floresta Ombrófila Densa/ } \\
\text { Campo Rupestre }\end{array}$ & $\begin{array}{l}\text { Lima Duarte, Olaria, Rio } \\
\text { Preto e Santa Bárbara do } \\
\text { Monte Verde (MG) }\end{array}$ & $900-1.670$ & 109 & 16 & Abreu et al., 2011 \\
\hline $\begin{array}{l}\text { Parque Estadual de } \\
\text { Ibitipoca (Ibi) }\end{array}$ & $\begin{array}{l}\text { Floresta Atlântica } \\
\text { Floresta Ombrófila Densa/ } \\
\text { Campo Rupestre }\end{array}$ & $\begin{array}{l}\text { Santa Rita de Ibitipoca e } \\
\text { Lima Duarte (MG) }\end{array}$ & $1.200-1.784$ & 118 & 25 & Menini Neto et al., 2007 \\
\hline Mata do Baú (Bau) & $\begin{array}{c}\text { Cerrado } \\
\text { Floresta Estacional Semidecidu- } \\
\text { al/ Campo-cerrado }\end{array}$ & Barroso (MG) & $900-1.200$ & 44 & 5 & Menini Neto et al., 2004b \\
\hline $\begin{array}{l}\text { R. B. da Represa do } \\
\text { Grama (RdG) }\end{array}$ & $\begin{array}{c}\text { Floresta Attântica } \\
\text { Floresta Estacional Semidecidu- } \\
\text { al }\end{array}$ & Descoberto (MG) & 750 & 28 & 1 & Menini Neto et al., 2004a \\
\hline $\begin{array}{l}\text { Vitória da Conquista } \\
\text { (VdC) }\end{array}$ & $\begin{array}{l}\text { Caatinga/ Floresta Atlântica } \\
\text { Floresta Estacional Decidual } \\
\text { Montana (Mata de Cipó) }\end{array}$ & Vitória da Conquista (BA) & $600-1.100$ & 44 & 16 & $\begin{array}{l}\text { Marinho; Azevedo, 2011; } \\
\text { 2013; Azevedo; Marinho, } \\
\text { 2012; Santos, } 2013\end{array}$ \\
\hline $\begin{array}{l}\text { Parque Nacional de } \\
\text { Boa Nova (BoN) }\end{array}$ & $\begin{array}{l}\text { Caatinga/Floresta Atlântica } \\
\text { Floresta Estacional Decidual } \\
\text { Montana (Mata de Cipó) }\end{array}$ & Boa Nova (BA) & $440-1.111$ & 42 & 5 & Rêgo; Azevedo, 2017 \\
\hline
\end{tabular}


Tabela 2. Lista de gêneros e espécies de Orchidaceae registradas em Vitória da Conquista, Bahia, Brasil. * $=$ novos registros para o estado da Bahia; $* *=$ novos registros para a região Nordeste do Brasil. Incluindo material testemunho (Vouchers) e herbário onde o material encontra-se depositado.

\begin{tabular}{|c|c|c|}
\hline Gêneros & Espécies & Vouchers \\
\hline \multirow[t]{4}{*}{ Acianthera } & Acianthera cristata (Barb.Rodr.) Luer & Marinho et al. 15 (HUESBVC) \\
\hline & Acianthera nemorosa (Barb.Rodr.) F.Barros & Azevedo et al. 405 (HVC) \\
\hline & Acianthera panduripetala (Barb.Rodr.) Pridgeon \& M.W. Chase** & Azevedo 1137 (HVC) \\
\hline & Acianthera saurocephala (Lodd.) Pridgeon \& M.W. Chase & Macbado \& Santos 963 (HUEFS) \\
\hline Anathallis & Anathallis pusilla (Barb.Rodr.) F.Barros & Azevedo et al. 416 (HVC) \\
\hline Barbosella & Barbosella crassifolia (Edwall) Schltr. & Marinbo et al. 65 (HUESBVC) \\
\hline Brassavola & Brassavola tuberculata Hook & Azevedo et al. 388 (HVC) \\
\hline \multirow[t]{4}{*}{ Campylocentrum } & Campylocentrum crassirbizum Hoehne & Marinho \& Azevedo 15 (HUESBVC) \\
\hline & Campylocentrum ornithorrbynchum (Lindl.) Rolfe & Arevedo et al. 424 (HVC) \\
\hline & Campylocentrum robustum Cogn. & Azevedo et al. 350 (HUEFS) \\
\hline & Campylocentrum sellowii (Rchb. f.) Rolfe & Azevedo et al. 389 (HVC) \\
\hline Campanemia & Campanemia micromera Barb.Rodr. & Machado 964 (ICN) \\
\hline Catasetum & Catasetum purum Nees \& Sinning & Azevedo et al. 604 (HVC) \\
\hline Cattleya & Cattleya amethystoglossa Linden \& Rchb.f. & Soares-Filho 1029 (HUESBVC) \\
\hline Cyclopogon & Cyclopogon variegatus Barb.Rodr. & Azrevedo et al. 380 (HVC) \\
\hline Cyrtopodium & Cyrtopodium bolstii L.C. Menezes & Azevedo et al. 390 (HVC) \\
\hline Dryadella & Dryadella aviceps (Rchb. f.) Luer & Azevedo et al. 605 (HVC) \\
\hline Eltroplectris & Eltroplectris triloba (Lindl.) Pabst & Marinbo et al. 68 (HUESBVC) \\
\hline \multirow[t]{3}{*}{ Encyclia } & Encyclia alboxantbina Fowlie & Dittrich 1586 (HUEFS) \\
\hline & Encyclia oncidioides (Lindl.) Schltr. & Mori 11296 (CEPEC) \\
\hline & Encyclia patens Hook & Azevedo et al. 438 (HVC) \\
\hline Epidendrum & Epidendrum densiflorum Hook & Azevedo et al. 437 (HVC) \\
\hline \multirow[t]{4}{*}{ Gomesa } & Gomesa ciliata (Lindl.) M.W.Chase \& N.H.Williams & Marinho \& Azevedo 24 (HUESBVC) \\
\hline & Gomesa nitida (Barb.Rodr.) M.W.Chase \& N.H.Williams & Toscano $2124(\mathrm{RB})$ \\
\hline & Gomesa ramosa (Lindl.) M.W.Chase \& N.H.Williams & Marinho et al. 526 (HUEFS) \\
\hline & Gomesa ranifera (Lindl.) M.W.Chase \& N.H.Williams & Azevedo 393 (HVC) \\
\hline Mesadenella & Mesadenella cuspidata (Lindl.) Garay & Azevedo et al. 423 (HVC) \\
\hline Miltonia & Miltonia flavescens (Lindl.) Lindl. & Azevedo 978 (HVC) \\
\hline Notylia & Notylia pubescens Lindl. & Azevedo et al. 401 (HVC) \\
\hline Oeceoclades & Oeceoclades maculata (Lindl.) Lindl. & Marinho et al. 2 (HUESBVC) \\
\hline \multirow[t]{3}{*}{ Pabstiella } & Pabstiella acrogenia Luer \& Toscano & Toscano de Brito 2317 (HUEFS) \\
\hline & Pabstiella quasi Luer \& Toscano & Toscano de Brito 1586 (HUEFS) \\
\hline & Pabstiella seriata (Lindl.) Luer \& Toscano* & Azevedo et al. 386 (HVC) \\
\hline Polystachya & Polystacbya concreta (Jacq.) Garay \& Sweet & Marinbo \& Azevedo 27 (HUESBVC) \\
\hline Prescottia & Prescottia stachyodes (Sw.) Lindl. & Marinho et al. 3 (HUESBVC) \\
\hline Psilocbilus & Psilocbilus modestus Barb. Rodr. & Marinho \& Azevedo 14 (HUESBVC) \\
\hline Sacoila & Sacoila lanceolata (Aubl.) Garay & Azevedo et al. 590 (HVC) \\
\hline Sarcoglottis & Sarcoglottis curvisepala Szlach. \& Rutk. & Marinho et al. 123 (HUESBVC) \\
\hline Specklinia & Specklinia bymenantha (Lindl.) F.Barros \& V.T.Rodrigues** & Azevedo et al. 385 (HVC) \\
\hline Stelis & Stelis aprica Lindl. & Marinho et al. 1 (HUESBVC) \\
\hline Trichocentrum & Trichocentrum pumilum (Lindl.) M.W. Chase \& N.H. Williams & Marinbo et al. 7 (HUESBVC) \\
\hline Vanilla & Vanilla chamissonis Klotzsch & Azevedo 959 (HVC) \\
\hline & Vanilla palmarum (Salzm. ex Lindl.) Lindl. & Azevedo et al. 384 (HVC) \\
\hline Zygostates & Zygostates kublmannii Brade & Azevedo et al. 404 (HVC) \\
\hline
\end{tabular}


Figura 1. Espécies representando os gêneros de Orchidaceae ocorrentes em Vitória da Conquista, Bahia, Brasil. a. Acianthera panduripetala (Barb.Rodr.) Pridgeon \& M.W. Chase. b. Anathallis pusilla (Barb.Rodr.) F.Barros. c. Barbosella crassifolia (Edwall) Schltr. d. Brassavola tuberculata Hook. e. Campylocentrum ornithorrhynchum (Lindl.) Rolfe. f. Capanemia micromera Barb.Rodr. g. Catasetum purum Nees \& Sinning. h. Cattleya amethystoglossa Linden \& Rchb.f. i. Cyclopogon variegatus Barb.Rodr. j. Cyrtopodium holstii L.C. Menezes. k. Dryadella aviceps (Rchb. f.) Luer. 1. Eltroplectris triloba (Lindl.) Pabst. (Fotos: C.O.Azevedo)
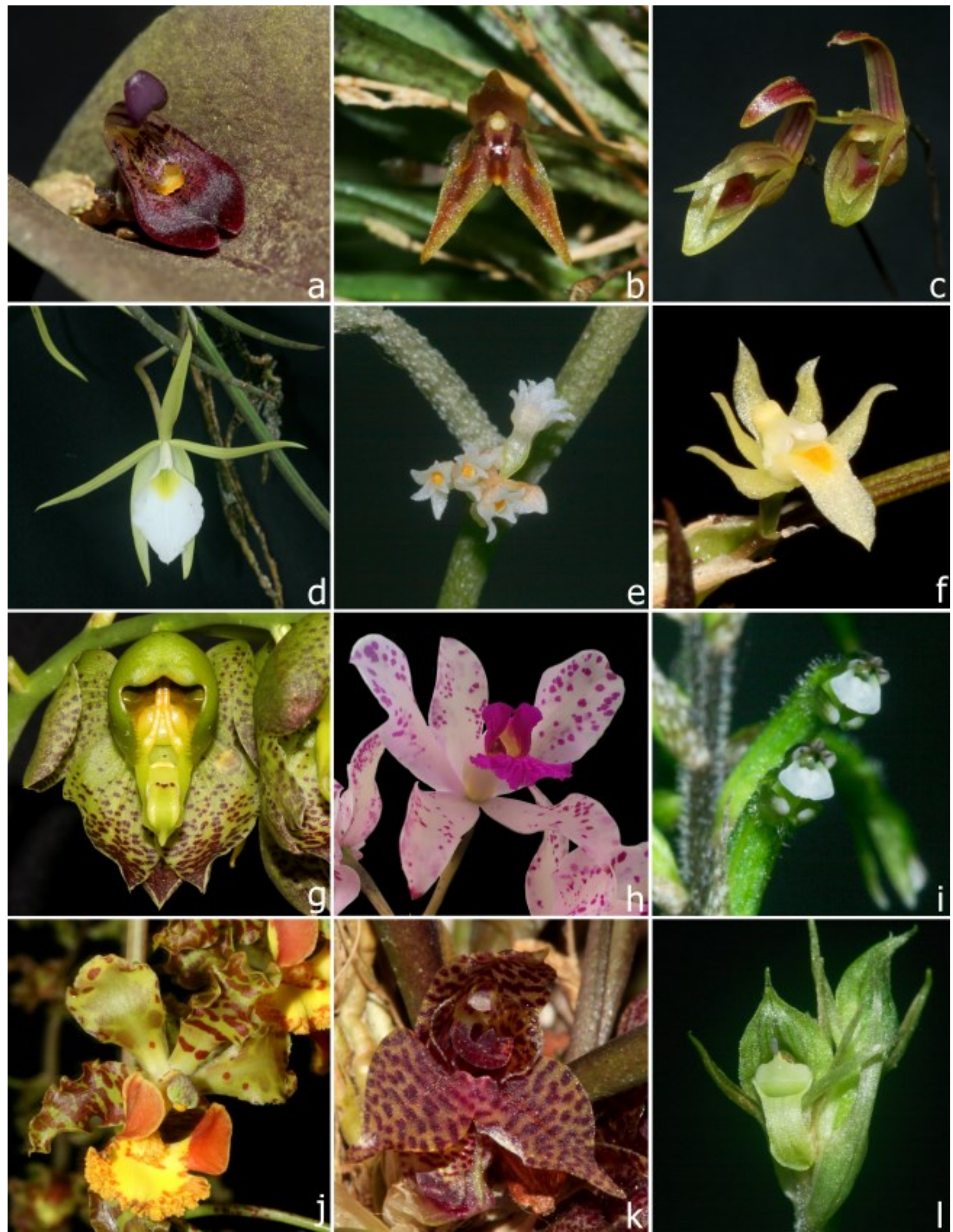
Figura 2. Espécies representando os gêneros de Orchidaceae ocorrentes em Vitória da Conquista, Bahia, Brasil. a. Encyclia alboxanthina Fowlie. b. Epidendrum densiflorum Hook. c. Gomesa ranifera (Lindl.) M.W.Chase \& N.H.Williams. d. Mesadenella cuspidata (Lindl.) Garay. e. Miltonia flavescens (Lindl.) Lindl. f. Notylia pubescens Lindl. g. Oeceoclades maculata (Lindl.) Lindl. h. Pabstiella seriata (Lindl.) Luer \& Toscano. i. Polystachya concreta (Jacq.) Garay \& Sweet. j. Prescottia stachyodes (Sw.) Lindl. k. Psilochilus modestus Barb. Rodr. 1. Sacoila lanceolata (Aubl.) Garay. (Fotos: C.O.Azevedo)
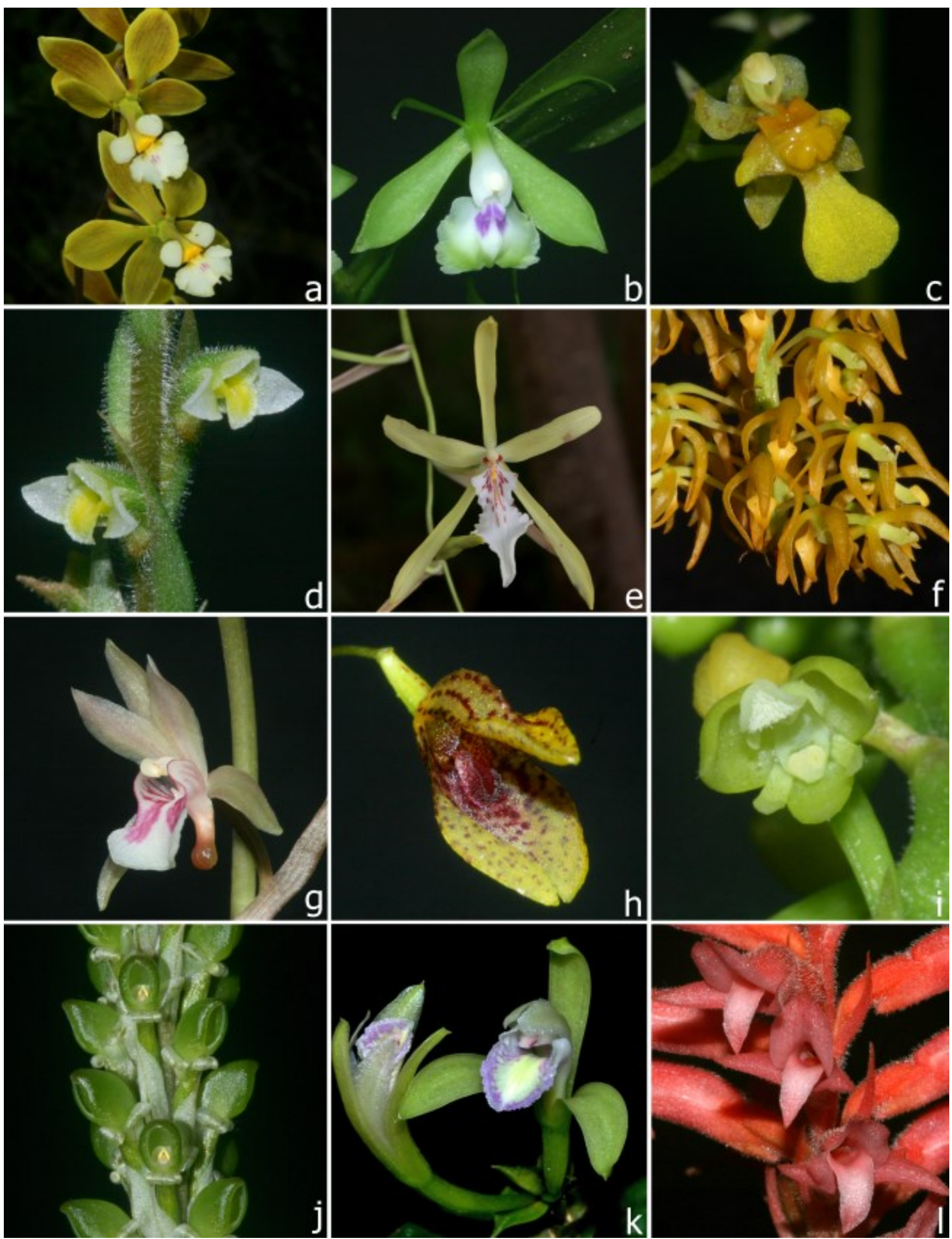
Figure 3. Espécies representando os gêneros de Orchidaceae ocorrentes em Vitória da Conquista, Bahia, Brasil. a. Sarcoglottis curvisepala Szlach. \& Rutk. b. Specklinia bymenantha (Lindl.) F.Barros \& V.T.Rodrigues. c. Stelis aprica Lindl. d. Trichocentrum pumilum (Lindl.) M.W. Chase \& N.H. Williams. e. Vanilla chamissonis Klotzsch. f. Zygostates kublmannii Brade. (Fotos: C.O.Azevedo)
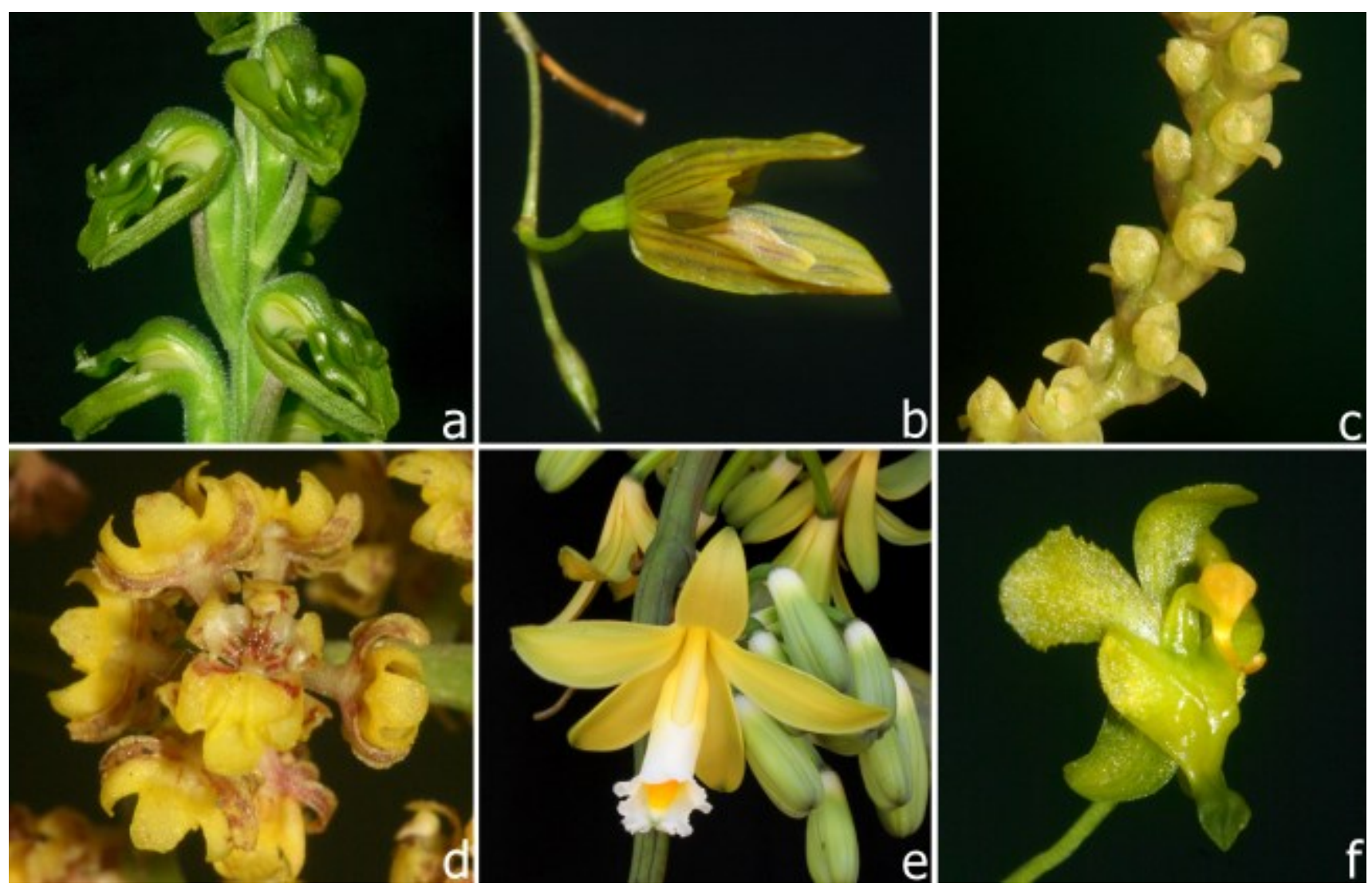

Rchb.f. ex R.Warner (Figura 2h - Cruz et al., 2003), não haviam sido registras para Vitória da Conquista, sendo este, em ambos os casos, o registro mais à oeste no estado da Bahia e o primeiro registro em áreas de Floresta Estacional.

A Serra de Grão-Mogol (GrM) foi a primeira área a divergir. Esta área é reconhecida pelo alto grau de endemismo e flora única. De forma semelhante, a análise de correspondência (Figura 4b) mostra claramente que Grão Mogol (GrM) é a área mais distinta. Em seguida, dois grupos foram formados (Figura 4a): o grupo A, agrupando as áreas de florestas secas (Floresta Estacional Decidual e Semidecidual) da Bahia e de Minas Gerais; e um grande grupo (D) que inclui as áreas de Campos Rupestres e de Floresta Ombrófila dos dois estados. Esses dois grupos têm a maioria das áreas distribuídas em diferentes domínios.

No grupo A, Vitória da Conquista $(\mathrm{VdC})$ aproximou-se da área do Parque Nacional de Boa Nova $(\mathrm{BoN})$, compondo o grupo $\mathrm{B}$ que foi recuperado como grupo-irmão do clado composto pelas áreas secas de Minas Gerais (grupo C): Reserva Biológica da Represa do Grama (RdG) + Mata do Baú (Bau). O grupo A é composto por áreas de florestas secas e de moderada altitude da Bahia e de Minas Gerais, demonstrando que entre os clados maiores não há correlação entre a distância e os agrupamentos, os quais direcionam para a aproximação de áreas com semelhanças ambientais. Vitória da Conquista (VdC) faz parte deste grupo e, junto ao Parque Nacional de Boa Nova (BoN), compõe o grupo B. Neste caso, além das semelhanças ambientais, a distância pode ter sido fator preponderante para o agrupamento. Ambos, $\mathrm{VdC}$ e $\mathrm{BoN}$, apresentam áreas de mata-de-cipó, um tipo de Floresta Estacional Decidual Montana, onde ocorrem várias espécies de ampla distribuição, como: Campylocentrum sellowii (Rchb.f.) Rolfe, \& N.H.Williams, Campylocentrum crassirbizum Hoehne, Gomesa ciliata (Lindl.) M.W.Chase, Notylia pubescens Lindl., Oeceoclades maculata (Lindl.) Lindl., Sacoila lanceolata (Aubl.) Garay, Trichocentrum pumilum (Lindl.) M.W. Chase \& N.H.Williams e Vanilla palmarum (Salzm. ex Lindl.) Lindl. Essas áreas estão situadas em uma região transicional entre a Caatinga e a Floresta Atlântica favoráveis à ocorrência de espécies de ambos os tipos vegetacionais. Entretanto, os valores de bootstrap indicam o fraco padrão de agrupamento das áreas do Sudoeste da Bahia (VdC + BoN) (apenas 42\% BS) e a análise de correspondência (Figura 4b) demonstra que a flora dessas áreas tem muitos elementos distintos. A flora da região deve ser estudada com mais detalhes, a fim de refinar o conhecimento sobre suas afinidades biogeográficas.

No grande grupo D foi possível identificar dois subgrupos: as duas áreas de Campo Rupestre se agruparam (grupo E), incluindo a Chapada Diamantina $(\mathrm{ChD})+$ Serra do Cipó (Cip), enquanto o outro grupo $\mathrm{F}$ engloba as áreas de mata úmida da Bahia e de Minas Gerais. Sendo que Serra Negra e do Funil (SNF) e o Parque Estadual de Ibitipoca (Ibi) são mais relacionados entre si do que com as áreas do Sul da Bahia (SdB) (Figura 4a). 
Os Campos Rupestres da Cadeia do Espinhaço estão, de forma geral, situados em áreas de transição entre o Cerrado, a Caatinga e a Floresta Atlântica, já os Campos de Altitude das Serras do Mar e da Mantiqueira encontram-se totalmente inseridos na região da Floresta Atlântica (Harley, 1995). Apesar de distintos, e estarem em domínios fitogeográficos diferentes, Campos Rupestres e Campos de Altitude apresentem semelhanças fisionômicas e compartilham algumas espécies de plantas (Vasconcelos, 2011). A distribuição disjunta entre plantas de áreas de Campos Rupestres e de Campos de Altitude associados à Floresta Atlântica foi encontrada para outros grupos de plantas (Giulietti; Pirani, 1988; Calió et al., 2008). Já a sugerida rela- ção entre as áreas de Campos Rupestres da Cadeia do Espinhaço devido, especialmente, a algumas espécies com padrões de distribuição entre o Planalto de Conquista e a Chapada Diamantina, não foi comprovado para Orchidaceae. Exceto por Encyclia alboxanthina Fowlie, as demais espécies documentadas em Vitória da Conquista estão distribuídas por outras áreas. Os valores de bootstrap indicam o fraco padrão de agrupamento das áreas de Campo Rupestre da Bahia e de Minas Gerais (grupo E, Cip + ChD), com 49\% BS, também demonstrado pela análise de correspondência, evidenciando que as áreas têm muitos elementos distintos.

Figura 4. Dendrograma mostrando a similaridade florística entre as dez áreas de florestas secas e úmidas da Bahia e Minas Gerais, utilizando o coeficiente de Dice com UPGMA sobre dados de presença e ausência de espécies de Orchidaceae. O suporte interno foi calculado utilizando 5.000 replicações de bootstrap. À direita, mapa de distribuição geográfica das áreas incluídas na análise. b. Eixos 1 e 2 de uma análise de correspondência sobre dados de presença e ausência de espécies de Orchidaceae entre dez áreas de florestas secas e úmidas da Bahia e Minas Gerais. Legenda: Bau (Mata do Baú), BoN (Parque Nacional de Boa Nova), ChD (Chapada Diamantina), Cip (Serra do Cipó), GrM (Serra de Grão-Mogol), Ibi (Parque Estadual de Ibitipoca), RdG (R. B. da Represa do Grama), SdB (Sul da Bahia), SNF (Serra Negra e do Funil), VdC (Vitória da Conquista).
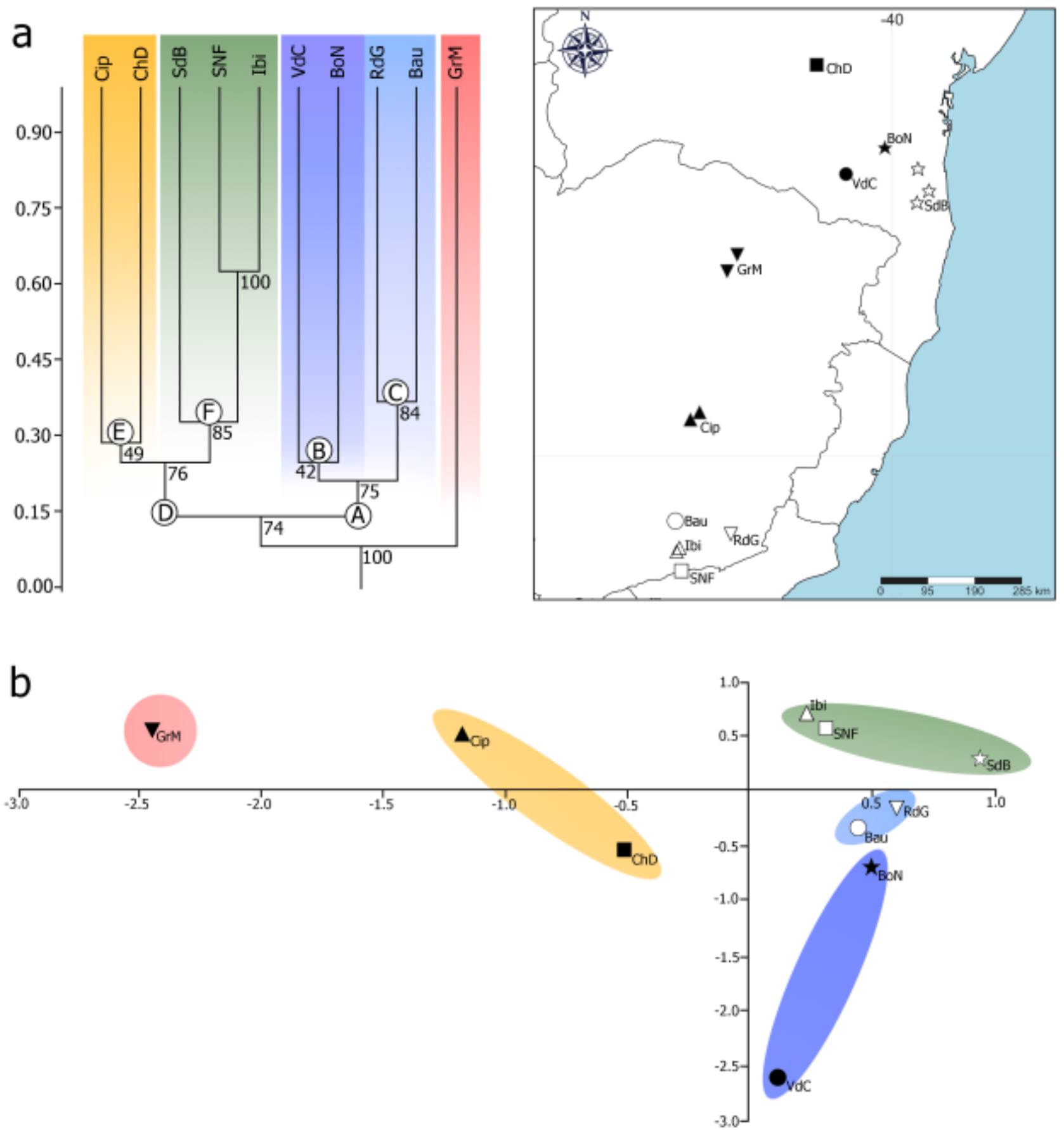
O grupo formado pela Serra Negra e do Funil (SNF) e o Parque Estadual de Ibitipoca (Ibi) apresentou os maiores valores de similaridade e bootstrap (ca. 0,62 - Dice; 100\% BS). Além disso, a análise de correspondência também indicou uma boa coesão entre essas áreas úmidas de Minas Gerais. Nesse caso, ambas representam áreas de Floresta Atlântica, compostas por Floresta Ombrófila Densa e formações de Campo Rupestre.

\section{Conclusão}

A presença de 44 espécies de orquídeas nas matas-de-cipó do município de Vitória da Conquista, sendo duas delas novas ocorrências para o Nordeste brasileiro e uma nova ocorrência para o estado da Bahia, revelam a importância dessa região para o conhecimento e a preservação da flora da Bahia. A lista atualizada das espécies de Orchidaceae de Vitória da Conquista permitiu testar as relações florísticas entre essa importante área do Planalto de Conquista e outras próximas que compartilham características geomorfológicas e fitofisionômicas. Embora haja muitas espécies com distribuição compartilhada entre Vitória da Conquista e a Chapada Diamantina, as análises de agrupamento mostraram uma maior aproximação entre as matasde-cipó e as florestas estacionais semideciduais de Minas Gerais. É possível que outros grupos taxonômicos encontrem outros resultados, mas, para isso, ainda é necessário investir em expedições de campo e formação de pessoal para conhecer ainda mais a vegetação do município e do Planalto de Conquista.

\section{Agradecimentos}

Agradecemos à Universidade Estadual do Sudoeste da Bahia (UESB) pelo apoio logístico e financeiro. À Toscano de Brito e a Edlley Pessoa pelo auxílio na identificação das espécies de Pleurothallidinae e Campylocentrum, respectivamente. À Aline Souza e a Anderson Machado pela companhia durante as coletas.

\section{Financiamento}

Esta pesquisa foi financiada pela Fundação de Amparo à Pesquisa do Estado da Bahia (FAPESB), por meio do projeto de pesquisa "Estudo taxonômico das espécies de Orchidaceae ocorrentes no município de Vitória da Conquista e arredores e criação de banco de DNA total da Universidade Estadual do Sudoeste da Bahia"; pela Universidade Estadual do Sudoeste da Bahia (UESB), por meio do projeto de pesquisa "Levantamento florístico das Orchidaceae da Caatinga do munićpio de Vitória da Conquista, Bahia, Brasil"; pelo Conselho Nacional de Desenvolvimento Científico e Tecnológico (CNPq), por meio da bolsa de iniciação científica concedida a LCM e MCS.

\section{Contribuição de Autoria}

Conceitualização: COA. Curadoria de dados: COA, LCM, MCS. Análise formal: COA Aquisição de financiamento: COA. Investigação: COA, LCM, MCS. Metodologia: COA, LCM, MCS. Administração do projeto: COA. Recursos: COA. Programas: COA. Supervisão: COA, LCM. Validação: COA, LCM, MCS. Visualização: COA LCM. Redação - rascunho original: COA, LCM. Redação - revisão e edição: COA, LCM, MCS. Produção das imagens: LCM.

\section{Conflito de Interesse}

Os autores declaram não haver conflitos de interesse a informar.

\section{Disponibilidade dos Dados}

Os dados integrais analisados durante o estudo atual podem ser fornecidos mediante solicitação justificada à autora para correspondência.

\section{Conformidade ética}

Não se aplica.

\section{Referências}

Abreu IS, Giulietti AM. Flora da Bahia: Aristolochiaceae. Sitientibus série Ciências Biológicas 2016;16:1-25 doi: $\underline{10.13102 /}$ $\underline{\text { scb1059 }}$

Abreu NL, Menini-Neto L, Konno TUP. Orchidaceae das Serras Negra e do Funil, Rio Preto, Minas Gerais, e similaridade florística entre formações campestres e florestais do Brasil. Acta Botanica Brasilica 2011;25(1):58-70. doi: 10.1590/S0102$\underline{33062011000100009}$

Albuquerque MA, Silva EL, Barros KNNO, Xavier Júnior SFA. Comparação entre coeficientes similaridade um aplicação em ciências florestais. Matemática e Estatística em Foco 2016;4 (2):102-114. Disponível em http://www.seer.ufu.br/index.php/ matematicaeestatisticaemfoco/article/view/26550 (acesso em 8 maio 2021).

Alves M, Roque N. Flora da Bahia: Asteraceae - Tribo Heliantheae. Sitientibus série Ciências Biológicas 2016;16:1-63. doi: $\underline{10.13102 / \mathrm{scb} 1127}$

Amorim A, Jardim JG, Lopes MMM, Fiaschi P, Borges RAX, Perdiz RO, Thomas WW. Angiospermas em remanescentes de floresta montana no sul da Bahia, Brasil. Biota Neotropica 2009;9 (3):313-348. doi: 10.1590/S1676-06032009000300028

Andrade-Lima D. Vegetação da área Jaguaquara-Maracás, Bahia. Ciência e Cultura 1971; 23(3):317-319.

Azevedo CO, Marinho LC, Machado AFP, Arroyo F, VázquezGarcía J. Magnolia brasiliensis (Magnoliaceae), a new species and new record for the Northeastern region of Brazil. Brittonia 2018;70(3):306-311. doi: 10.1007/s12228-018-9529-1

Azevedo CO, Marinho LC. Novos registros de Orchidaceae para o Nordeste brasileiro: Acianthera tricarinata e Cyclopogon variegatus. Sitientibus série Ciências Biológicas 2012;12(2):339-344. doi: $10.13102 / \mathrm{scb} 147$

Azevedo CO, van den Berg C. A família Orchidaceae no Parque Municipal de Mucugê, Bahia, Brasil. Hoehnea 2007;34(1):1-47. doi: $\underline{10.1590 / \text { S2236-89062007000100001 }}$

Barros F, Pinheiro F. Flora de Grão-Mogol, Minas Gerais: Orchidaceae. Boletim de Botânica 2004;22(2):361-383. doi: $10.11606 /$ issn.2316-9052.v22i2p361-383

Barros F. Orchidaceae. In: Giulietti AM, Menezes NL, Pirani JR, Meguro M, Wanderley MGL. Flora da Serra do Cipó, Minas Gerais: caracterização e lista de espécies. Boletim de Botânica 1987;9:1-151. doi: 10.11606/issn.2316-9052.v9i0p1-151

Bastos CA, van den Berg C. A Família Orchidaceae no município de Morro do Chapéu, Bahia, Brasil. Rodriguésia 2012a;63(4):883 -927. doi: 10.1590/S2175-78602012000400010

Bastos CA, van den Berg, C. Flora da Bahia: Catasetum (Orchidaceae). Sitientibus série Ciências Biológicas 2012b;12: 83 -89. DOI: http://dx.doi.org/10.13102/scb113

Calió MF, Pirani JR, Struwe L. Morphology-based phylogeny and revision of Prepusa and Senaea (Gentianaceae: Helieae): — rare endemics from eastern Brazil. Kew Bulletin 2008;63(2):169-191. doi: $10.1007 / \mathrm{s} 12225-008-9030-1>$

Castro RM, Rapini A. Four new species of Moraceae from Bahia, Brazil. Neodiversity 2006;1(2):13-20. doi: 10.13102/NEOD.12.2

Coelho MM, Amorim AM. Floristic composition of the Montane Forest in the Almadina-Barro Preto axis, Southern Bahia, Brazil. Biota Neotropica 2014;14(1):1-41. doi: 10.1590/S1676$\underline{06033878}$

Cruz DTC, Borba EL; van den Berg C. O gênero Cattleya Lindl. (Orchidaceae) no Estado da Bahia, Brasil. Sitientibus série Ciências Biológicas 2003;3(1/2):26-34.

Davis JC. Statistics and data analisys in geology. New York: John Wiley \& Sons; 1986. 
Dittrich VAO, Souza AM. Additions to the fern flora of Northeastern Brazil. Ernstia 2013;23(2):119-123.

Flora do Brasil. Orchidaceae. In: Flora do Brasil $2020 \mathrm{em}$ construção. Jardim Botânico do Rio de Janeiro. Disponível em http:// reflora.jbri.gov.br/reflora/floradobrasil/FB179 (acesso em 10 fev 2021).

Giulietti AM, Pirani J R. Patterns of geographic distribution of some plant species from the Espinhaço range, Minas Gerais and Bahia, Brazil. In: Vanzolini PE, Heyer, HR (eds.) Proceedings of a workshop on neotropical distribution patterns. Rio de Janeiro: Academia Brasileira de Ciências; 1988. p.39-69.

Giulietti AM, Queiroz LP, Silva TRS, França F, Guedes ML, Amorim AM. Flora da Bahia. Sitientibus série ciências biológicas 2006;6(3):169-173.

Goldenberg R, Jesus JC, Amorim AM. Miconia babiana (Melastomataceae, Miconieae), a new species from semideciduous forest in Bahia, Brazil. Plant Ecology and Evolution 2020;153(1):152-159. doi: 10.5091/plecevo.2020.1659

Hammer $\varnothing$, Harper DAT, Ryan PD. PAST: Paleontological statistics software package for education and data analysis. Palaeontologia Electronica 2001;4(1):1-9.

Harley RM, Simmons NA. Florula of Mucugê: Chapada Diamantina - Bahia, Brazil. Kew: Royal Botanic Gardens; 1986.

Harley RM. Introduction. In: Stannard BL, Harvey YB, Harley RM (eds.) Flora of the Pico das Almas, Chapada Diamantina - Bahia, Brazil. Kew: Royal Botanic Gardens; 1995. p.1-42.

Leitman P, Amorim A, Menini-Neto L, Forzza R. Epiphytic angiosperms in a mountain forest in southern Bahia, Brazil. Biota Neotropica 2014;14(2):1-12. doi: 10.1590/S1676$\underline{06032014001013}$

Leme EMC, Till W, Kollmann LJC, Moura RL, Ribeiro OBC. Miscellaneous New Species of Brazilian Bromeliaceae - III. Phytotaxa 2014;177(2):61-100. doi: 10.11646/phytotaxa.177.2.1

Leme EMC. A new Quesnelia Species from Bahia. Journal of the Bromeliad Society 2008;58(6):269.

Luer CA, Toscano de Brito ALV. Miscellaneous New Species and Combinations in the Pleurothallidinae (Orchidaceae) from Brazil and Argentina. Harvard Papers in Botany 2011;16(2):361-382. doi: $10.3100 / 0.25 .016 .0207$

Marinho LC, Azevedo CO. Acianthera saurocephala (Lodd.) Pridgeon \& M.W.Chase (Orchidaceae: Pleurothallidinae): novo registro para o Nordeste brasileiro. Revista Brasileira de Biociências 2011;9(4):554-557.

Marinho LC, Azevedo CO. Orchidaceae na Reserva do Poço Escuro, Vitória da Conquista, Bahia, Brasil. Sitientibus série ciências biológicas 2013;13:1-14. doi: 10.13102/scb213

Menini-Neto L, Almeida VR, Forzza RC. A família Orchidaceae na Reserva Biológica da Represa do Grama - Descoberto, Minas Gerais, Brasil. Rodriguésia 2004a;55(84):137-156. doi: 10.1590/2175-78602004558409

Menini-Neto L, Alves RJV, Barros F, Forzza RC. Orchidaceae do Parque Estadual de Ibitipoca, MG, Brasil. Acta Botanica Brasilica 2007;21(3):687-696. doi: 10.1590/S0102-33062007000300015

Menini-Neto L, Assis LCS, Forza RC. A família Orchidaceae em um fragmento de floresta estacional semidecidual no município de Barroso, Minas Gerais, Brasil. Lundiana 2004b;5(1):9-27.

Moura JN, Caires CS. A família Solanaceae Juss. no município de Vitória da Conquista, Bahia, Brasil. Paubrasilia 2021;4:e0049. doi: $\underline{10.33447 / \text { paubrasilia.2021.e0049 }}$

Queiroz LP, Conceição AA, Giulietti AM. Nordeste semi-árido: caracterização geral e lista das fanerógamas. In: Giulietti AM, Conceição AA, Queiroz LP. Diversidade e caracterização das fanerógamas do semi-árido brasileiro. Recife: Associação Plantas do Nordeste; 2006, p. 15-359.
Rêgo HT, Azevedo CO. Sinopse das Orchidaceae do Parque Nacional de Boa Nova, BA, Brasil. Hoehnea 2017;44(1):70-89. doi: 10.1590/2236-8906-44/2016.

Santos MA, Conceição LP, Pereira FA, Oliveira IB, Santos AKA. Desmidiaceae (Zygnematophyceae, Streptophyta) da Lagoa das Bateias, Vitória da Conquista, Bahia, Brasil. Sitientibus série Ciências Biológicas 2013;13:1-11. doi: 10.13102/scb226

Santos MC. Orchidaceae de um fragmento de floresta estacional na fazenda Oriente, Vitória da Conquista, Bahia, Brasil. Monografia [Graduação em Ciências Biológicas] - Universidade Estadual do Sudoeste da Bahia, Vitória da Conquista; 2013.

Silva-Castro MM, Costa CRA, Brito RF. Flora da Bahia - Bignoniaceae 1: Jacaranda Jussieu. Sitientibus série Ciências Biológicas 2007;7(1):15-31. Disponível on-line em http://www2.uefs.br/ revistabiologia/pg7 n1.html (acesso em 8 maio 2021).

Smidt EC, Toscano de Brito ALV. Checklist das Orchidaceae da Chapada Diamantina, Bahia, Brasil. In: Toscano de Brito ALV, Cribb P. As Orquídeas da Chapada Diamantina. Rio de Janeiro: Nova Fronteira; 2005. p. 278-285.

Sneath PHA, Sokal RR. Numerical taxonomy. San Francisco: W. H. Freeman; 1973.

Souza AM, Valente EB, Azevedo CO. Musgos de um fragmento de floresta estacional semidecidual do município de Vitória da Conquista, Bahia, Brasil. Pesquisas, Botânica 2015;67:217-233.

Taylor NP. Plants in peril, 17: Melocactus conoideus. The Kew Magazine 1992;9(3):138-142. doi: 10.1111/j.1467-8748.1992.tb00086.x

Thiers B. Index Herbariorum. Part I: The herbaria of the world. New York Botanical Garden. Disponível em: http:/ / sweetgum.nybg.org/science/ih/ (acesso em 28 out 2020).

Toscano-de-Brito ALV, Queiroz LP. Orchidaceae. In: Zappi DC, Lucas E, Stannard BL, Lughadha EN, Pirani JR, Queiroz LP, Atkins S, Hind DJN, Giulietti AM, Harley RM, Carvalho AM. Lista das plantas vasculares de Catolés, Chapada Diamantina, Bahia, Brasil. Boletim de Botânica da Universidade de São Paulo 2003;21(2):396-397. doi: 10.11606/issn.2316-9052.v21i2p345$\underline{398}$

Toscano-de-Brito ALV. Orchidaceae. In: Guedes MLS, Orge MD. Checklist das espécies vasculares do Morro do Pai Inácio (Palmeiras) e Serra da Chapadinha (Lençóis), Chapada Diamantina, Bahia, Brasil. Salvador: Universidade Federal da Bahia; 1998. p. 53-54.

Toscano-de-Brito ALV. Orchidaceae. In: Stannard BL. Flora of the Pico das Almas: Chapada Diamantina, Bahia, Brazil. Kew: Royal Botanic Gardens; 1995. p. 725-767.

Vasconcelos MF. O que são campos rupestres e campos de altitude nos topos de montanha do Leste do Brasil? Revista Brasileira de Botânica 2011;34(2):241-246. doi: 10.1590/S0100$\underline{84042011000200012}$

Vieira TL, Barros F, Roque N. Orchidaceae no município de Jacobina, estado da Bahia, Brasil. Hoehnea 2014;41(3):469-482. 10.1590/S2236-89062014000300012

\section{Como citar este artigo} How to cite this article

\section{(ABNT)}

AZEVEDO, C. O.; SANTOS, M. C.; MARINHO, L. C. Orchidaceae no município de Vitória da Conquista, Bahia: lista de espécies e similaridade florística entre áreas da Bahia e Minas Gerais. Paubrasilia, Porto Seguro, v. 4, e0065, 2021. DOI 10.33447/ paubrasilia.2021.e0065

\section{(Vancouver)}

Azevedo CO, Santos MC, Marinho LC. Orchidaceae no município de Vitória da Conquista, Bahia: lista de espécies e similaridade florística entre áreas da Bahia e Minas Gerais. Paubrasilia 2021;4:e0065. doi: 10.33447/paubrasilia.2021.e0065 